\title{
Interacting Dark Fluids in LRS Bianchi Type-II Universe
}

\author{
Vijay Gulabrao Meteㄹ, Pratiksha Brahmadeo Murade², Abhijit Shankarrao Bansod ${ }^{3}$ \\ ${ }^{1}$ Department of Mathematics, R. D. I. K. \& K. D. College, Amravati, India \\ ${ }^{2}$ Department of Applied Science, PRMIT \& R, Amravati, India \\ ${ }^{3}$ Department of Applied Science, V. Y. W. S. Polytechnic, Badnera, India \\ Email:vmete5622@gmail.com, pratikshamurade@gmail.com, bansodabhijit@yahoo.com
}

How to cite this paper: Mete, V.G., Murade, P.B. and Bansod, A.S. (2018) Interacting Dark Fluids in LRS Bianchi Type-II Universe. Open Access Library Journal, 5: e4900.

https://doi.org/10.4236/oalib.1104900

Received: September 12, 2018

Accepted: October 28, 2018

Published: October 31, 2018

Copyright (C) 2018 by authors and Open Access Library Inc.

This work is licensed under the Creative

Commons Attribution International

License (CC BY 4.0).

http://creativecommons.org/licenses/by/4.0/

\section{cc) (i) Open Access}

\begin{abstract}
At the present paper, Locally Rotationally Symmetric (LRS) Bianchi type-II cosmological model with interacting dark matter (DM) and holographic dark energy (DE) have been discussed. In order to obtain solutions of the field equations, it is assume that the shear scalar $(\sigma)$ is proportional to expansion scalar $(\theta)$. To have a general description of holographic dark energy and dark matter, a phenomenological parameterization of dark energy in terms of its equation of state (EoS) has been taken. Statefinder diagnostic pair i.e. $\{r, s\}$ is adopted to separate other existing dark energy models from this model. Here we discuss two models: when $n=1 / 2$, we obtain acyclic universe and the model converges into phantom region whereas when $n=3 / 2$, we get a expanding universe and the model converges into quintessence region. Some important geometrical and physical features regarding to this model have also been studied.
\end{abstract}

\section{Subject Areas}

Particle Physics

\section{Keywords}

LRS Bianchi Type-II Space-Time, Interacting Dark Fluids, Statefinder Parameters

\section{Introduction}

The phase of accelerating expansion of the universe is indicated by Type Ia Supernovae (SNeIa) [1], [2], the Sloan Digital Sky Survey (SDSS) (Seljack et al. [3]) and Wilkinson Microwave Anisotropy Probe (WMAP) [4]. The astrophysical 
observation, also indicates some evidence that cosmic media not a perfect fluid [5]. The mysterious term, known as dark energy, occupying about $73 \%$ of dark energy of the universe, is considered to be responsible for its acceleration. The remaining configuration of energy of the universe is composed of $23 \%$ of dark matter and $4 \%$ of baryonic matter. The easiest candidate for DE is cosmological constant $(\Lambda)$ [6], which is used to explain the observed cosmological investigations.

During the evolution of the universe, EoS parameter can be used to differentiate several dark energy models in standard cosmological model. To fit different properties of dark energy many candidates have been proposed such as tachyon [7]; k-essence [8]; phantom [9], [10]; quintom [11]; ghost condensate [12]; Brane world models [13]; interacting dark energy models [14]; chaplygin gas models [15]; holographic dark energy models [16]; phantom scalar field [17]. Cosmological models like coupled dark energy, holographic dark energy, $F(R, T)$ gravity, $F(T)$ gravity, $F(R)$ gravity, scalar field theory and $\Lambda \mathrm{CDM}$ cosmological models represent the increasing expansion with phantom/quintessence nature with cosmography tests have been studied by Bamba et al. [18]. In the framework of different matrices, exact general relativistic cosmological models in context of DE in presence of anisotropic fluid have been investigated (Yadav and Yadav [19]; Kumar and Yadav [20]; Reddy et al. [21]; Kumar and Akarsu [22]; Naidu et al. [23]; Akarsu and Kiling, [24]; Adhav et al. [25]; Katore et al. [26]). In the literature, anisotropic Bianchi type-I, III \& V dark energy models in presence of usual perfect fluid have also been investigated (Yadav et al. [27]; Akarsu and Kiling [28]; Singh and Chaubey [29]; Pradhan [30]; Yadav and Shah [31]; Saha and Yadav [32]).

According to some basic quantum gravitational principle, nature of dark energy known as holographic dark energy can be studied. This principle [33] also states that the degree of freedom in a bounded system must be finite and doesn't scale by its volume but with its boundary era. Here $\rho_{\Lambda}$ means vacuum energy density. With the help of cosmology, we consider $\rho_{\Lambda}$ as DE density. Hooft [34] consider this holographic principle in context of black hole physics, which is another alternative to the solution of DE problem. In purview of dark energy problem, however, holographic principle put forwards a relation between Hubble parameter and Holographic dark energy density as $\rho_{\Lambda}=H^{2}$. Present accelerated expansion of the universe doesn't given by this value. The form of holographic density is given as $\rho_{\Lambda} \approx \alpha H^{2}+\beta \dot{H}$ in which $H$ denote Hubble parameter, $\alpha$ and $\beta$ are constant. This was imposed by Granda and Olivers [35], which must satisfy current observational data.For the purpose of reconstruction of holographic scalar field models of the dark energy, numbers of works have been done.

An important role in the early stage of the universe was played by anisotropy thereby making it necessary to study the homogeneous as well as the anisotropic cosmological models. Therefore the models based on Bianchi types universe are assumed to be spatially homogeneous models of cosmology which are in general 
anisotropic. The correspondences between the holographic dark energy and each one of phantom, tachyon, chaplygin gas and generalized chaplygin gas in FRW universe have been investigated by Setare [36] [37] [38] [39]. Cosmological dynamics of interacting holographic dark energy model have been pointed out by Setare and Venegus [40]. Bianchi type space-time having holographic dark energy's evolution with the help of constant deceleration parameter has been shown by Sarkar and Mahanta [41]. For instant, Sarkar [42] [43] [44] have studied holographic dark energy model in Bianchi typesspace-time with linearly varying deceleration parameter in general relativity. Also Adhav et al. [45] [46], Kiran et al. [47] and Reddy et al. [48] have investigated holographic DE in Bianchi models.

The geometric parameters Hubble parameter $(H)$ and deceleration parameter (q) were duly defined for characterizing the expansion history of the universe. From the observation of the cosmic acceleration $q<0$, it can be stated that $\mathrm{DE}$ with negative equation of state (EoS) $\omega<-1 / 3$ and likely $\omega \sim-1$ exist. Leading to the need of higher time derivative of scale factor $a$, new term $\dddot{a}$ is introduced for the deduction of the informational context regarding the dynamical evolution of $\omega$. Sahni et al. [49] defined a new parameter called as statefinder parameter as

$$
r=\frac{\dddot{a}}{a H^{3}}, \quad s=\frac{r-1}{3(q-1 / 2)}
$$

Note that $r$ also known as cosmic jerk. The geometry describe by the set of quantities extended to include $\{h, q, r, s\}$. Different cosmological models corresponding to trajectories in the $s-r$ plane shows quantitatively different behaviors. Fixed point $\left.\{s, r\}\right|_{\Lambda \mathrm{CDM}}=\{1,0\}$ corresponds to a spatially flat $\Lambda \mathrm{CDM}$ (cosmological constant $\Lambda$ with cold dark matter) scenario. A better way of establishing the "distance" from a given point of departure of dark energy to the fixed point from spatially flat $\Lambda \mathrm{CDM}$ was provide in this model, can be demonstrated from [49] [50]. For differentiation of variety of dark energy involvingcosmological constant, interacting dark energy model, Brane world model, the chaplygin gas, quintessence, quintom, phantom, use of statefinder parameters may be successful (Sahni et al. [49]; Alam et al. [50]; Zimdahl and Pavon [51]; Zhang [52]). With the help of $r(s)$ evolution diagram, it is very easy to identify "distance" the flat $\Lambda$ CDM scenario to the given dark energy model.

At the present paper, the LRS Bianchi type-II cosmological modelwith interacting dark matter (DM) and holographic dark energy (DE) are investigated. Statefinder diagnostic pair i.e. $\{r, s\}$ is adopted to separate other existing dark energy models from this model. Some important geometrical and physical features of the models have also been discussed.

The physical parameters of cosmological importance for LRS Bianchi type-II space-time are:

The volume scale factor:

$$
V=a_{1}^{2} a_{2}
$$


The average scale factor:

$$
a=\left(a_{1}^{2} a_{2}\right)^{\frac{1}{3}}
$$

The mean Hubble parameter:

$$
H=\frac{1}{3} \frac{\dot{V}}{V}
$$

The deceleration parameter:

$$
q=\frac{\mathrm{d}}{\mathrm{d} t}\left(\frac{1}{H}\right)-1
$$

The mean anisotropy parameter:

$$
\Delta=\frac{1}{3} \sum_{i=1}^{3}\left(\frac{H_{i}-H}{H}\right)^{2}
$$

where $H_{1}=H_{3}=\frac{\dot{a}_{1}}{a_{1}}, H_{2}=\frac{\dot{a}_{1}}{a_{1}}$ be the directional Hubble parameters along $x, y, z$ axes respectively.

\section{Metric and Field Equations}

The spatially homogeneous and anisotropic LRS Bianchi type-IImetric can be written as

$$
\mathrm{d} s^{2}=-\mathrm{d} t^{2}+a_{1}^{2} \mathrm{~d} x^{2}+a_{2}^{2}(\mathrm{~d} y-x \mathrm{~d} z)^{2}+a_{1}^{2} \mathrm{~d} z^{2}
$$

where $a_{1}$ and $a_{2}$ are the scale factors and functions of the cosmic time $t$ only.

The Einstein's field equations in GR given as $(8 \pi G=1$ and $c=1)$

$$
R_{i j}-\frac{1}{2} g_{i j} R=-\left({ }^{m} T_{i j}+{ }^{\wedge} T_{i j}\right)
$$

Also, matter tensor for cold dark matter (pressureless i.e. $w_{m}=0$ ) and holographic dark energy are given as

$$
\begin{gathered}
{ }^{m} T_{i j}=\rho_{m} u_{i} u_{j} \\
{ }^{\Lambda} T_{i j}=\left(\rho_{\Lambda}+p_{\Lambda}\right) u_{i} u_{j}+g_{i j} p_{\Lambda}
\end{gathered}
$$

where $\rho_{m}$ denote the energy density of dark matter, $\rho_{\Lambda}$ represent the energy density and $p_{\Lambda}$ is the pressure of holographic dark energy.

The Einstein's field Equation (2.2) for LRS Bianchi type-II model (2.1) with the help of matter tensor (2.3) to (2.4) can be written as

$$
\begin{gathered}
\frac{2 \ddot{a}_{1}}{a_{1}}+\frac{\dot{a}_{1}^{2}}{a_{1}^{2}}-\frac{3 a_{2}^{2}}{4 a_{1}^{4}}=-p_{\Lambda} \\
\frac{\ddot{a}_{1}}{a_{1}}+\frac{\ddot{a}_{2}}{a_{2}}+\frac{\dot{a}_{1} \dot{a}_{2}}{a_{1} a_{2}}+\frac{a_{2}^{2}}{4 a_{1}^{4}}=-p_{\Lambda} \\
2 \frac{\dot{a}_{1} \dot{a}_{2}}{a_{1} a_{2}}+\frac{\dot{a}_{1}^{2}}{a_{1}^{2}}-\frac{a_{2}^{2}}{4 a_{1}^{4}}=\rho_{\Lambda}+\rho_{m}
\end{gathered}
$$

where overhead dot denotes derivative with respect to cosmic time $t$. 
Conservation equations stating the interaction between the DM and DE as,

$$
\begin{gathered}
\dot{\rho}_{m}+\left(\frac{\dot{V}}{V}\right) \rho_{m}=Q \\
\dot{\rho}_{\Lambda}+\left(\frac{\dot{V}}{V}\right)\left(1+w_{\Lambda}\right) \rho_{\Lambda}=-Q
\end{gathered}
$$

$w_{\Lambda}$ represents the state parameter for holographic dark energy and is given by $w_{\Lambda}=\frac{p_{\Lambda}}{\rho_{\Lambda}}$.

The quantity $Q$ indicates for the interacting term. The direction of transfer of energy depends upon the sign of $Q$. We consider that $Q$ be positive, which stands for energy transfer from dark energy to dark matter. If $Q$ is negative, stands for energy transfer from dark matter to dark energy. Overhead dot denotes the derivative with respect to commoving time.These continuity equations state that interacting term should be a function of a quantity with unit inverse of time i.e. $Q \propto \frac{1}{t}$ (Hubble parameter is the first and natural choice) multiplied with energy density. The conservation equation and other models stating the interaction between DE and DM were established by Wetterich [53] [54] (see also Billyard and Coley [55]) and were used side by side the holographic DE by Horvat [56]. Expression for interaction between dark energy (DE) and dark matter (DM) can be written phenomenological as (Cai and Wang [57]; Amendola et al. [58]; Guo et al. [59] [60]).

$$
Q=3 b^{2} H \rho_{m}=b^{2} \frac{\dot{V}}{V} \rho_{m}
$$

where $b^{2}$ represents a coupling constant.

We obtain the energy density of DM from equations (2.8) and (2.9) as

$$
\rho_{m}=\rho_{0} V^{\left(b^{2}-1\right)}
$$

where $\rho_{0}>0$ is a real constant of integration.

Using Equations (2.10) and (2.11), we get the interacting term as

$$
Q=3 \rho_{0} b^{2} H V^{\left(b^{2}-1\right)}
$$

\section{Cosmological Solutions}

It may be simply observed that the system have three equations as Equations (2.5)-(2.7) having five unknowns $a_{1}, a_{2}, \rho_{m}, \rho_{\Lambda}$ and $p_{m}$. Therefore to get set of solutions, one extra probable relation among these parameters needed. For this we considered that expansion scalar $(\theta)$ proportional to the shear scalar $(\sigma)$. This gives

$$
a_{1}=m a_{2}^{n}
$$

where $a_{1}$ and $a_{2}$ are the metric potentials and $n>0, m>0$ are constants. Thorne [61] explained the intension behind considering this condition. The universe's Hubble parameter is isotropy in present day within $\approx 30$ percent (Kan- 
towski and Sachs [62]; Kristian and Sachs [63]). This has been suggested by the reviews of the velocity-red-shift relation for extra lactic sources. To place accurately, red-shift studies space the limit

$$
\frac{\sigma}{H} \leq 0.3
$$

on the proportion of shear $(\sigma)$ to Hubble parameter $(H)$ in neighborhood of galaxy at present day. Usual congruence to the homogeneous expansion persuades that the relation $\frac{\sigma}{\theta}$ remain invariant, this has been noted by Collins et al. [64].

From Equations (2.5) and (2.6), we get,

$$
\frac{\dot{a}_{1} \dot{a}_{2}}{a_{1} a_{2}}-\frac{\ddot{a}_{1}}{a_{1}}+\frac{\ddot{a}_{2}}{a_{2}}+\frac{a_{2}^{2}}{a_{1}^{4}}-\frac{\dot{a}_{1}^{2}}{a_{1}^{2}}=0
$$

Equation (3.2) with the help of by Equation (3.1) leads to

$$
\frac{\ddot{a}_{2}}{a_{2}}+2 n\left(\frac{\dot{a}_{2}^{2}}{a_{2}^{2}}\right)+\frac{a_{2}^{2-4 n}}{(1-n) m^{4}}=0, \quad n \neq 1,
$$

which reduces to

$$
2 \ddot{a}_{2}+4 n\left(\frac{\dot{a}_{2}^{2}}{a_{2}}\right)=\frac{2 a_{2}^{3-4 n}}{(n-1) m^{4}}, \quad n \neq 1
$$

On simplification Equation (3.3) convert to

$$
\frac{\mathrm{d}}{\mathrm{d} a_{2}}\left(\phi^{2}\right)+\frac{4 n}{a_{2}} \phi^{2}=\frac{2 a_{2}^{3-4 n}}{(n-1) m^{4}}
$$

where $\dot{a}_{2}^{2}=\phi\left(a_{2}\right), \quad \ddot{a}_{2}=\phi \phi^{\prime}, \quad \phi^{\prime}=\frac{\mathrm{d} \phi}{\mathrm{d} a_{2}}$.

From Equation (3.4), we get

$$
\phi^{2}=\dot{a}_{2}^{2}=\frac{a_{2}^{4-4 n}}{2(n-1) m^{4}}+k a_{2}^{-4 n}
$$

where $k>0$ be real integrating constant.

Equation (3.5) leads to

$$
\frac{a_{2}^{2 n} \mathrm{~d} S}{\sqrt{\frac{a_{2}^{4}}{2 m^{4}(n-1)}+k}}=\mathrm{d} t
$$

The solution of (3.6) is not tenable for $n=1$. One can choose the value of $n$ such that the above relation can be integrable. We consider the following two cases.

First Model for $n=\frac{1}{2}$

When $n=\frac{1}{2}$, Equation (3.6) reduces to

$$
\frac{a_{2} \mathrm{~d} a_{2}}{\sqrt{k-\frac{a_{2}^{4}}{m^{4}}}}=\mathrm{d} t
$$

which after integration and $k=1$, leads to 


$$
a_{2}=m \sqrt{\sin \left(\frac{2\left(t+c_{1}\right)}{m^{2}}\right)}
$$

where $c_{1}$ is constant of integration. Equation (3.13) for $n=\frac{1}{2}$, leads to

$$
a_{1}=m^{3 / 2} \sqrt[4]{\sin \left(\frac{2\left(t+c_{1}\right)}{m^{2}}\right)}
$$

Therefore, the metric (3) reduces to

$$
\begin{aligned}
\mathrm{d} s^{2}= & -\mathrm{d} t^{2}+m^{3} \sqrt{\sin \left(\frac{2\left(t+c_{1}\right)}{m^{2}}\right)} \mathrm{d} x^{2}+m^{2} \sin \left(\frac{2\left(t+c_{1}\right)}{m^{2}}\right)(\mathrm{d} y-x \mathrm{~d} z)^{2} \\
& +m^{3} \sqrt{\sin \left(\frac{2\left(t+c_{1}\right)}{m^{2}}\right)} \mathrm{d} z^{2}
\end{aligned}
$$

The volume scale factor, average scale factor, mean Hubble parameter, deceleration parameter, shear scalar and mean anisotropy parameter obtained as,

$$
\begin{gathered}
V=m^{4} \sin \left(\frac{2\left(t+c_{1}\right)}{m^{2}}\right) \\
a=\left(m^{4} \sin \left(\frac{2\left(t+c_{1}\right)}{m^{2}}\right)\right)^{1 / 3} \\
H=\frac{2}{3 m^{2}} \cot \left(\frac{2\left(t+c_{1}\right)}{m^{2}}\right) \\
q=-1+\frac{3}{m^{2}} \sec ^{2}\left(\frac{2\left(t+c_{1}\right)}{m^{2}}\right) \\
\Delta=\frac{1}{8}
\end{gathered}
$$

Using Equation (3.11) in Equations (2.11) and (2.12), we get energy density of dark matter and interacting term as,

$$
\begin{gathered}
\rho_{m}=\rho_{0} m^{4\left(b^{2}-1\right)} \sin ^{\left(b^{2}-1\right)}\left(\frac{2\left(t+c_{1}\right)}{m^{2}}\right) \\
Q=2 b^{2} \rho_{0} m^{2\left(2 b^{2}-3\right)} \sin ^{\left(b^{2}-2\right)}\left(\frac{2\left(t+c_{1}\right)}{m^{2}}\right) \cos \left(\frac{2\left(t+c_{1}\right)}{m^{2}}\right)
\end{gathered}
$$

With the help of Equations (3.7), (3.8) and (2.18) in Equation (2.7), we get the energy density of holographic dark energy as,

$$
\rho_{\Lambda}=\frac{5 \cot ^{2}\left(\frac{2\left(t+c_{1}\right)}{m^{2}}\right)-1}{4 m^{4}}-\rho_{0} m^{4\left(b^{2}-1\right)} \sin ^{\left(b^{2}-1\right)}\left(\frac{2\left(t+c_{1}\right)}{m^{2}}\right)
$$

With the help of Equations (3.7), (3.8) in Equation (2.6), we get the pressure of holographic dark energy as, 


$$
p_{\Lambda}=\frac{5 \cot ^{2}\left(\frac{2\left(t+c_{1}\right)}{m^{2}}\right)+11}{4 m^{4}}
$$

Using Equations (3.18) and (3.19), the EoS parameter of holographic dark energy is obtain as,

$$
\begin{gathered}
w_{\Lambda}=\frac{p_{\Lambda}}{\rho_{\Lambda}} \\
w_{\Lambda}=\frac{5 \cot ^{2}\left[\frac{2\left(t+c_{1}\right)}{m^{2}}\right]+11}{\frac{4 m^{4}}{5 \cot ^{2}\left[\frac{2\left(t+c_{1}\right)}{m^{2}}\right]-1}-\rho_{0} m^{4\left(b^{2}-1\right)} \sin ^{\left(b^{2}-1\right)}\left[\frac{2\left(t+c_{1}\right)}{m^{2}}\right]}
\end{gathered}
$$

Statefinder parameters is defined and given as,

$$
r=\frac{\dddot{a}}{a H^{3}}, \quad s=\frac{r-1}{3(q-1 / 2)}, \quad s=\frac{2(r-1)}{r-11}
$$

Second Model for $n=\frac{3}{2}$

When $n=\frac{3}{2}$, Equation (3.6) reduces to

$$
\frac{a_{2}^{3} \mathrm{~d} S}{\sqrt{\frac{a_{2}^{4}}{m^{4}}+k}}=\mathrm{d} t
$$

which after integration leads to

$$
a_{2}=\left(\frac{4}{m^{4}}\left(t+k_{1}\right)^{2}-m^{4} k\right)^{\frac{1}{4}}
$$

where $c_{1}$ is constant of integration. Equation (3.1) for $n=\frac{3}{2}$, leads to

$$
a_{1}=m\left(\frac{4}{m^{4}}\left(t+k_{1}\right)^{2}-m^{4} k\right)^{\frac{3}{8}}
$$

Therefore, the metric (2.1) reduces to

$$
\begin{aligned}
\mathrm{d} s^{2}= & -\mathrm{d} t^{2}+m^{2}\left(\frac{4}{m^{4}}\left(t+k_{1}\right)^{2}-m^{4} k\right)^{\frac{3}{4}} \mathrm{~d} x^{2} \\
& +\frac{16}{m^{2}}\left(\frac{4}{m^{4}}\left(t+k_{1}\right)^{2}-m^{4} k\right)^{\frac{1}{2}}(\mathrm{~d} y-x \mathrm{~d} z)^{2} \\
& +m^{2}\left(\frac{4}{m^{4}}\left(t+k_{1}\right)^{2}-m^{4} k\right)^{\frac{3}{4}} \mathrm{~d} z^{2}
\end{aligned}
$$

The volume scale factor, average scale factor, mean Hubble parameter, deceleration parameter, shear scalar and mean anisotropy parameter are obtained as, 


$$
\begin{gathered}
V=m^{2}\left(\frac{4}{m^{4}}\left(t+k_{1}\right)^{2}-m^{4} k\right) \\
a=m^{2 / 3}\left(\frac{4}{m^{4}}\left(t+k_{1}\right)^{2}-m^{4} k\right)^{2 / 3} \\
H=\frac{8\left(t+k_{1}\right)}{3\left[4\left(t+k_{1}\right)^{2}-m^{8} k\right]} \\
q=\frac{1}{2}+\frac{3 m^{8} k}{8\left(t+k_{1}\right)^{2}} \\
A_{m}=\frac{1}{32}
\end{gathered}
$$

Using Equation (3.27) in Equations (2.11) and (2.12), we get energy density of dark matter and interacting term as,

$$
\begin{gathered}
\rho_{m}=\rho_{0} m^{-2}\left[4\left(t+k_{1}\right)^{2}-m^{8} k\right]^{\left(b^{2}-1\right)} \\
Q=8 b^{2} \rho_{0}\left(t+k_{1}\right) m^{-2\left(b^{2}-1\right)}\left[4\left(t+k_{1}\right)^{2}-m^{8} k\right]^{\left(b^{2}-1\right)}
\end{gathered}
$$

With the help of Equations (3.24), (3.25) and (3.32) in the Equation (3.2.7), the energy density of holographic dark energy obtain as,

$$
\begin{aligned}
\rho_{\Lambda}= & \frac{21\left(t+k_{1}\right)^{2}}{\left[4\left(t+k_{1}\right)^{2}-m^{8} k\right]^{2}}-\frac{1}{4\left[4\left(t+k_{1}\right)^{2}-m^{8} k\right]} \\
& -\rho_{0} m^{-2}\left[4\left(t+k_{1}\right)^{2}-m^{8} k\right]^{\left(b^{2}-1\right)}
\end{aligned}
$$

With the help of Equations (3.24), (3.25) in the linear combination of Equations (2.5)-(2.6), the pressure of holographic dark energy obtained as,

$$
p_{\Lambda}=\frac{21\left(t+k_{1}\right)^{2}}{\left[4\left(t+k_{1}\right)^{2}-m^{8} k\right]^{2}}-\frac{21}{4\left[4\left(t+k_{1}\right)^{2}-m^{8} k\right]}
$$

Using Equations (3.34) and (3.35), the EoS parameter of holographic dark energy is obtain as,

$$
\begin{gathered}
w_{\Lambda}=\frac{p_{\Lambda}}{\rho_{\Lambda}} \\
w_{\Lambda}=\frac{\frac{21\left(t+k_{1}\right)^{2}}{\left[4\left(t+k_{1}\right)^{2}-m^{8} k\right]^{2}}-\frac{21}{4\left[4\left(t+k_{1}\right)^{2}-m^{8} k\right]}}{\frac{21\left(t+k_{1}\right)^{2}}{\left[4\left(t+k_{1}\right)^{2}-m^{8} k\right]^{2}}-\frac{1}{4\left[4\left(t+k_{1}\right)^{2}-m^{8} k\right]}-\rho_{0} m^{-2}\left[4\left(t+k_{1}\right)^{2}-m^{8} k\right]^{\left(b^{2}-1\right)}}
\end{gathered}
$$

Statefinder parameters is defined as

$$
r=\frac{\dddot{a}}{a H^{3}}=1+\frac{9 m^{8} k}{4\left(t+k_{1}\right)^{2}}
$$




$$
s=\frac{r-1}{3(q-1 / 2)}=2
$$

\section{Discussion}

Here we discussed two models for $n=1 / 2$ and $n=3 / 2$. In the model $n=1 / 2$, as time increases spatial volume also increases. As $t \rightarrow 0, V \rightarrow m^{4} \sin \left(\frac{2 c_{1}}{m^{2}}\right)$ and as $t \rightarrow \infty, V \rightarrow \infty$. Accelerating expansion of the model occurs for the situation $\sec ^{2}\left(\frac{2\left(t+c_{1}\right)}{m^{2}}\right)<\frac{m^{2}}{3}$. Whereas it is simply observe that model is in state of deceleration is obtained for the situation when $\sec ^{2}\left(\frac{2\left(t+c_{1}\right)}{m^{2}}\right)>\frac{m^{2}}{3}$. It is important to note that the model (3.10) include periodic function which is responsible for cyclic universe. Also in this model $\omega_{\Lambda}$ starts from phantom region and it reaches to $\omega_{\Lambda}=1$ (i.e. cosmological constant). After some finite time it enter into quintessence region $\left(-1<\omega_{\Lambda}<-1 / 3\right)$ and again come back into phantom region. Model for $n=3 / 2$, as $t \rightarrow 0, V \rightarrow$ constant and as $t \rightarrow \infty, V \rightarrow \infty$ i.e. universe expands as time increases. As $t \rightarrow 0, \omega_{\Lambda} \rightarrow 0$, and as time increases $\omega_{\Lambda}$, starts from phantom region. For some finite time, it extended to $\omega_{\Lambda}=-1$ (i.e. cosmological constant), which denotes that our model drive to $\Lambda \mathrm{CDM}$. After some time, it reaches to quintessence region $\left(-1<\omega_{\Lambda}<-1 / 3\right)$ for late time as shown in Figure 1. The available observation in cosmology, especially the three year WMAP data (Sprengel et al. [65]), the SNeIa data (Riess et al. [66]; Asteir et al. [67]) and the SDSS data (Eisenstein et al. [68]) pointed out that the $\Lambda \mathrm{CDM}$ model or the model reducible to $\Lambda \mathrm{CDM}$ are present as a standard model in cosmology, which is similar to present universe. Figure 2 shows that the $s-r$ plane having evolving trajectory for corresponding model is different from other dark energy models.

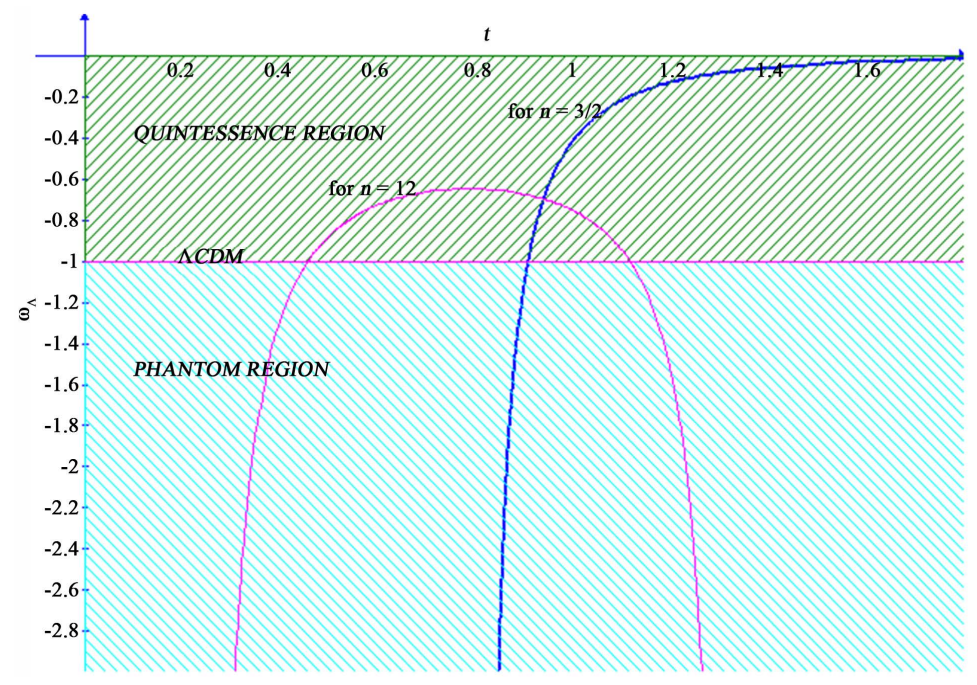

Figure 1. Evolution of EoS parameter $\omega_{\Lambda}$ versus cosmic time t. 


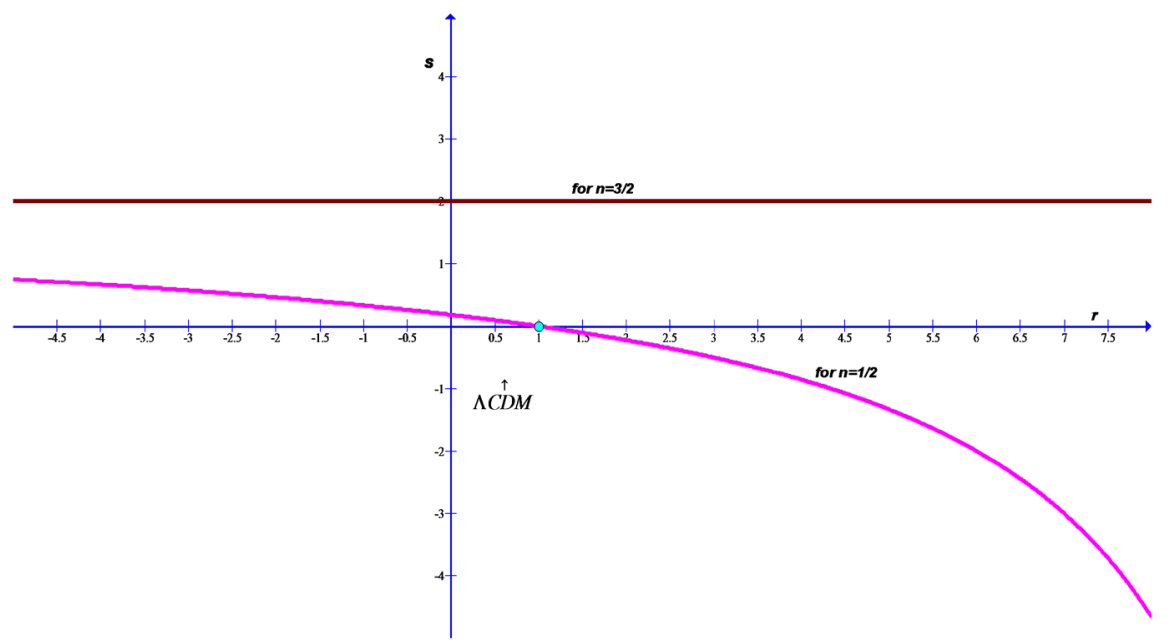

Figure 2. State finder parameters s versus $r$.

\section{Conclusion}

In this paper, we have studied LRS Bianchi Type-II universe filled with interacting dark matter and holographic dark energy. Here we have discussed two models for 1$) n=1 / 2$;2) $n=3 / 2$. In the model $n=1 / 2$, we obtained the cyclic universe and in case of model for $n=3 / 2$, we obtained the expanding universe. The cosmological parameter matches with the present day observations. The model for $n=1 / 2$ converges into phantom region whereas the model for $n=3 / 2$ converges into quintessence region. The statefinder parameter $\{r, s\}$ has been calculated in order to differentiate our model of dark energy with all other models of dark energy. We aspire that the future accurate observation will be capable of determining these statefinder parameters and consequently explore the nature of DE.

\section{Acknowledgements}

The author would like to acknowledge his deep sense of gratitude to the anonymous referee whose valuable comments and suggestion have improved this work.

\section{Conflicts of Interest}

The authors declare no conflicts of interest regarding the publication of this paper.

\section{References}

[1] Perlmutter, S. (1999) Measurements of $\Omega$ and $\Lambda$ from 42 High-Redshift Supernovae. The Astrophysical Journal, 517, 565-586. https://doi.org/10.1086/307221

[2] Riess, A.G., Filippenko, A.V., et al. (1998) Observational Evidence from Supernovae for an Accelerating Universe and a Cosmological Constant. The Astronomical Journal, 116, 1009-1032. https://doi.org/10.1086/300499

[3] Seljack, U., Makarov, A., et al. (2005) Cosmological Parameter Analysis including 
SDSS Ly-Alpha Forest and Galaxy Bias: Constraints on the Primordial Spectrum of Fluctuations, Neutrino Mass, and Dark Energy. Physical Review Journals, D71, Article ID: 103515.

[4] Bennett, C.L., Halpern, M., Hinshaw, G., Jarosik, N., Kogut, A., et al. (2003) First year Wilkinson Microwave Anisotropy Probe (WMAP) Observations: Preliminary Maps and Basic Results. The Astrophysical Journal Supplement Series, 148, 1-27. https://doi.org/10.1086/377253

[5] Jaffe, R.L. and Wilczek, F. (2003) Diquarks and Exotic Spectroscopy. Physical Review Letters, 91, Article ID: 232003. https://doi.org/10.1103/PhysRevLett.91.232003

[6] Hawkins Ed., Maddox, S., Cole, S., Madgwick, D., Norberg, P., et al. (2003) The 2dF Galaxy Redshift Survey: Correlation Functions, Peculiar Velocities and the Matter Density of the Universe. Monthly Notices of the Royal Astronomical Society, 346, 78-96. https://doi.org/10.1046/j.1365-2966.2003.07063.x

[7] Liddle, A.R. and Scherer, R.J. (1998) Classification of Scalar Field Potentials with Cosmological Scaling Solutions. Physical Review D, 59, Article ID: 023509. https://doi.org/10.1103/PhysRevD.59.023509

[8] Caldwell, R.R., Dave, R. and Steinhardt, P.J. (1998) Cosmological Imprint of an Energy Component with General Equation of State. Physical Review Letters, 80, Article ID: 1582. https://doi.org/10.1103/PhysRevLett.80.1582

[9] Steinhardt, P.J., Wang, L.M. and Zlatev, I. (1999) Cosmological Tracking Solutions. Physical Review D, 59, Article ID: 123504.

https://doi.org/10.1103/PhysRevD.59.123504

[10] Dvali, G.R., Gabadadze, G. and Porrati, M. (2000) Metastable Gravitons and Infinite Volume Extra Dimensions. Physics Letters B, 484, 112-118. https://doi.org/10.1016/S0370-2693(00)00631-6

[11] Capozziello, S., Carloni, S. and Troisi, A. (2003) Quintessence without Scalar Fields. Astronomy and Astrophysics, 1, 625.

[12] Deffayet, L. (2001) Cosmology on a Brane in Minkowski Bulk. Physics Letters B, 502, 199-208. https://doi.org/10.1016/S0370-2693(01)00160-5

[13] Nojiri, S. and Odintsov, S.D. (2003) Modified Gravity with Negative and Positive Powers of Curvature: Unification of Inflation and Cosmic Acceleration. Physical Review D, 68, Article ID: 123512. https://doi.org/10.1103/PhysRevD.68.123512

[14] Carroll, S.M., et al. (2003) Is Cosmic Speed-Up Due to New Gravitational Physics? Physical Review D, 70, Article ID: 043528. https://doi.org/10.1103/PhysRevD.70.043528

[15] Townsend, P.K. and Wohlfarth, N.R. (2003) Accelerating Cosmologies from Compactification. Physical Review Letters, 91, Article ID: 061302. https://doi.org/10.1103/PhysRevLett.91.061302

[16] Maldacena, J.M. and Nunez, C. (2001) Supergravity Description of Field Theories on Curved Manifolds and a No Go Theorem. International Journal of Modern Physics A, 16, 822-855. https://doi.org/10.1142/S0217751X01003937

[17] Ohta, N. (2003) Accelerating Cosmologies from Spacelike Branes. Physical Review Letters, 91, Article ID: 061303. https://doi.org/10.1103/PhysRevLett.91.061303

[18] Bamba, K., et al. (2012) Dark Energy Cosmology: The Equivalent Description via Different Theoretical Models and Cosmography Tests. Astrophysics and Space Science, 342, 155-228. https://doi.org/10.1007/s10509-012-1181-8

[19] Yadav, A.K. and Yadav, L. (2010) Bianchi Type III Anisotropic Dark Energy Models with Constant Deceleration Parameter. International Journal of Theoretical Physics, 
50, 218-227.

[20] Kumar, S. and Yadav, A.K. (2011) Some Bianchi Type-V Models of Accelerating Universe with Dark Energy. Modern Physics Letters A, 26, 647-659. https://doi.org/10.1142/S0217732311035018

[21] Reddy, D.R.K., Satyanarayana, B. and Naidu, R.L. (2012) Five Dimensional Dark Energy Model in Scalar-Tensor Theory of Gravitation. Astrophysics and Space Science, 339, 401-404. https://doi.org/10.1007/s10509-012-1007-8

[22] Kumar, S. and Akarsu, O. (2012) Bianchi Type-II Models in the Presence of Perfect Fluid and Anisotropic Dark Energy. The European Physical Journal Plus, 127, 64. https://doi.org/10.1140/epjp/i2012-12064-4

[23] Naidu, R.L., et al. (2012) LRS Bianchi Type-II Dark Energy Model in a Scalar-Tensor Theory of Gravitation. Astrophysics and Space Science, 338, 333-336. https://doi.org/10.1007/s10509-011-0935-Z

[24] Akarsu, O. and Kiling, C.B. (2010) Bianchi Type III Models with Anisotropic Dark Energy. General Relativity and Gravitation, 42, 763-775.

https://doi.org/10.1007/s10714-009-0878-7

[25] Adhav, K.S., et al. (2011) Bianchi Type-V Cosmological Model with Linearly Varying Deceleration Parameter. International Journal of Mathematical Archive, 2, 2149-2156.

[26] Katore, S.D., et al. (2012) Dynamics of Kantowski-Sachs Universe with Magnetized Anisotropic Dark Energy. Astrophysics and Space Science, 337, 393-400. https://doi.org/10.1007/s10509-011-0826-3

[27] Yadav, A.K., Rahaman, F. and Ray, S. (2011) Dark Energy Models with Variable Equation of State Parameter. International Journal of Theoretical Physics, 50, 871-881. https://doi.org/10.1007/s10773-010-0628-3

[28] Akarsu, O. and Kiling, C.B. (2010) De Sitter Expansion with Anisotropic Fluid in Bianchi Type-I Space-Time. Astrophysics and Space Science, 326, 315-322. https://doi.org/10.1007/s10509-009-0254-9

[29] Singh, T. and Chaubey, R. (2008) Bianchi Type-I Universe with Wet Dark Fluid. Pramana, 71, 447-458. https://doi.org/10.1007/s12043-008-0124-y

[30] Pradhan, A., et al. (2011) Bianchi Type-I Anisotropic Dark Energy Model with Constant Deceleration Parameter. International Journal of Theoretical Physics, 50, 2923-2938. https://doi.org/10.1007/s10773-011-0793-Z

[31] Yadav, A.K. and Saha, B. (2012) LRS Bianchi-I Anisotropic Cosmological Model with Dominance of Dark Energy. Astrophysics and Space Science, 337, 759-765. https://doi.org/10.1007/s10509-011-0861-0

[32] Saha, B. and Yadav, A.K. (2012) Dark Energy Model with Variable $q$ and $\omega$ in LRS Bianchi-II Space-Time. Astrophysics and Space Science, 341, 651-656. https://doi.org/10.1007/s10509-012-1070-1

[33] Susskind, L. (1995) The World as a Hologram. Journal of Mathematical Physics, 36, 6377. https://doi.org/10.1063/1.531249

[34] Hooft, G. (1993) Dimensional Reduction in Quantum Gravity. General Relativity and Quantum Cosmology. arXiv:gr-qc/9310026

[35] Granda, L.N. and Oliveros, A. (2008) Infrared Cut-Off Proposal for the Holographic Density. Physics Letters B, 669, 275-277. https://doi.org/10.1016/j.physletb.2008.10.017

[36] Setare, M.R. (2007) Interacting Holographic Phantom. The European Physical Journal C, 50, 991-998. https://doi.org/10.1140/epjc/s10052-007-0262-2 
[37] Setare, M.R. (2007) Holographic Tachyon Model of Dark Energy. Physics Letters B, 653, 116-121. https://doi.org/10.1016/j.physletb.2007.08.011

[38] Setare, M.R. (2007) The Holographic Dark Energy in Non-Flat Brans-Dicke Cosmology. Physics Letters B, 644, 99-103. https://doi.org/10.1016/j.physletb.2006.11.033

[39] Setare, M.R. (2007) Interacting Holographic Generalized Chaplygin Gas Model. Physics Letters B, 654, 1-6. https://doi.org/10.1016/j.physletb.2007.08.038

[40] Setare, M.R. and Vanegas, E.C. (2009) The Cosmological Dynamics of Interacting Holographic Dark Energy Model. International Journal of Modern Physics D, 18, 147-157. https://doi.org/10.1142/S0218271809014303

[41] Sarkar, S. and Mahanta, C.R. (2013) Holographic Dark Energy Model with Quintessence in Bianchi Type-I Space-Time. International Journal of Theoretical Physics, 52, 1482-1489. https://doi.org/10.1007/s10773-012-1468-0

[42] Sarkar, S. (2014) Holographic Dark Energy Model with Linearly Varying Deceleration Parameter and Generalized Chaplygin Gas Dark Energy Model in Bianchi Type-I Universe. Astrophysics and Space Science, 349, 985-993. https://doi.org/10.1007/s10509-013-1684-y

[43] Sarkar, S. (2014) Holographic Dark Energy with Linearly Varying Deceleration Parameter and Escaping Big Rip Singularity of the Bianchi Type-V Universe. Astrophysics and Space Science, 352, 859-866. https://doi.org/10.1007/s10509-014-1920-0

[44] Sarkar, S. (2014) Interacting Holographic Dark with Variable Deceleration Parameter and Tachyon Scalar Field Dark Energy Model in LRS Bianchi Type-II Universe. Astrophysics and Space Science, 350, 821-829. https://doi.org/10.1007/s10509-014-1786-1

[45] Adhav, K.S., Tayde, G.B. and Bansode, A.S. (2014) Interacting Dark Matter and Holographic Dark Energy in an Anisotropic Universe. Astrophysics and Space Science, 353, 249-257. https://doi.org/10.1007/s10509-014-2015-7

[46] Adhav, K.S., Munde, S.L., Tayade, G.B. and Bokey, V.D. (2015) Interacting Dark Matter and Holographic Dark Energy in Bianchi Type-V Universe. Astrophysics and Space Science, 359, 24. https://doi.org/10.1007/s10509-015-2471-8

[47] Kiran, M., et al. (2015) Bianchi Type-III Minimally Interacting Holographic Dark Energy Model with Linearly Varying Deceleration Parameter in Brans-Dicke Theory. Astrophysics and Space Science, 360, 54. https://doi.org/10.1007/s10509-015-2563-5

[48] Reddy, D.R.K., et al. (2015) LRS Bianchi Type-II Cosmological Model with a Linearly Varying Deceleration Parameter in $\mathrm{f}(\mathrm{R}, \mathrm{T})$ Gravity. Prespacetime Journal, 6, 1100 .

[49] Sahni, V., Saini, T.D., Starobinsky, A.A. and Alam, U. (2003) Statefinder-A New Geometrical Diagnostic of Dark Energy. Journal of Experimental and Theoretical Physics Letters, 77, 201-206. https://doi.org/10.1134/1.1574831

[50] Alam, U., Sahni, V., Saini, T.D. and Starobinsky, A.A. (2003) Exploring the Expanding Universe and Dark Energy Using the Statefinder Diagnostic. Monthly Notices of the Royal Astronomical Society, 344, 1057-1074. https://doi.org/10.1046/j.1365-8711.2003.06871.x

[51] Zimdahl, W. and Pavon, D. (2004) Statefinder Parameters for Interacting Dark Energy. General Relativity and Gravitation, 36, 1483-1491. https://doi.org/10.1023/B:GERG.0000022584.54115.9e

[52] Zhang, X. (2005) Statefinder Diagnostic for Coupled Quintessence. Physics Letters 
B, 611, 1-7. https://doi.org/10.1016/j.physletb.2005.02.022

[53] Wetterich, C. (1988) Cosmology and the Fate of Dilatation Symmetry. Nuclear Physics B, 302, 668-696. https://doi.org/10.1016/0550-3213(88)90193-9

[54] Wetterich, C. (1995) The Cosmon Model for an Asymptotically Vanishing Time-Dependent Cosmological "Constant". Astronomy \& Astrophysics, 301, 321-328.

[55] Billyard, A.P. and Coley, A.A. (2000) Interactions in Scalar Field Cosmology. Physical Review D, 61, Article ID: 083503. https://doi.org/10.1103/PhysRevD.61.083503

[56] Horvat, R. (2004) Holography and a Variable Cosmological Constant. Physical Review $D$, 70, Article ID: 087301. https://doi.org/10.1103/PhysRevD.70.087301

[57] Cai, R.G. and Wang, A. (2005) Cosmology with Interaction between Phantom Dark Energy and Dark Matter and the Coincidence Problem. Journal of Cosmology and Astroparticle Physics, 2005, 002. https://doi.org/10.1088/1475-7516/2005/03/002

[58] Amendola, L., et al. (2007) Consequence of Dark Matter-Dark Energy Interaction on Cosmological Parameters Derived from Type Ia Supernova Data. Physical Review D, 75, Article ID: 083506. https://doi.org/10.1103/PhysRevD.75.083506

[59] Guo, Z.K., Ohta, N. and Tsujikawa, S. (2007) Probing the Coupling between Dark Components of the Universe. Physical Review D, 76, Article ID: 023508. https://doi.org/10.1103/PhysRevD.76.023508

[60] Guo, Z.K., Ohta, N. and Zhang, Y.Z. (2007) Parametrizations of the Dark Energy Density and Scalar Potentials. Modern Physics Letters A, 22, 883-890. https://doi.org/10.1142/S0217732307022839

[61] Thorne, K.S. (1967) Primordial Element Formation, Primordial Magnetic Fields, and Isotropy of the Universe. Astrophysical Journal, 148, 51-68.

https://doi.org/10.1086/149127

[62] Kantowski, R. and Sachs, R.K. (1966) Some Spatially Homogeneous Anisotropic Relativistic Cosmological Models. Journal of Mathematical Physics, 7, 433. https://doi.org/10.1063/1.1704952

[63] Kristian, J. and Sachs, R.K. (1966) Observations in Cosmology. Astrophysical Journal, 143, 379. https://doi.org/10.1086/148522

[64] Collins, C.B., Glass, E.N. and Wilkinson, D.A. (1980) Exact Spatially Homogeneous Cosmologies. General Relativity and Gravitation, 12, 805-823. https://doi.org/10.1007/BF00763057

[65] Spergel, D.N., et al. (2003) First-Year Wilkinson Microwave Anisotropy Probe (WMAP) Observations: Determination of Cosmological Parameter. Astrophysical Journal Supplement Series, 148, 175-194.

[66] Riess, A.G., Strolger, L.G., et al. (2004) Type Ia Supernovae Discoveries at from the Hubble Space Telescope: Evidence for Past Deceleration and Constraints on Dark Energy Evolution. Astrophysical Journal, 607, 665-687.

[67] Astier, P., Guy, J., Regnault, N., Pain, R., Aubourg, E., et al. (2006) The Supernova Legacy Survey: Measurement of $\Omega_{\mathrm{M}}, \Omega_{\Lambda}$ and from First Year Data Set. Astronomy and Astrophysics, 447, 31-48. https://doi.org/10.1051/0004-6361:20054185

[68] Eisenstein, D.J., Zehavi, I., Hogg, D.W., et al. (2005) Detection of Baryon Acoustic Peak in the Large-Scale Correlation Function of SDSS Luminous red Galaxies. $A s$ trophysical Journal, 633, 560-574. 